finding.

From above reasons subdural collection of fluid must be divided into three group.

\title{
n-7. Angiographic Study on the Acute Traumatic Intracranial Hematoma relating to the Operative Prognosis
}

\author{
Hiroki Hirai, Norio Mikami and Teruyoshi Hashiba \\ Department of Neurosurgery, Sapporo Medical College and Hospital
}

The pesent study was done to draw the attention to the correlation between the angiographic findings and the operative prognosis in our series of the acute traumatic intracranial hematoma. To decide the extent of the hematoma the following points were angiographically noticed.

1) Inanterioposterior view

a) Thickness of the avascular area

b) Falx sign

c) Change in A and B line (averas and Wood)

2) In lateral view

Change in the difference between $\mathrm{H}_{1}$ line and $\mathrm{H}_{2}$ line (Vlahovitch) in length

The result is follows.

1) The thickness of the avascular area has no correlation with the operative mortality in the epidural hematoma, while in the subdural hematoma the thicker avascular area, the higher mortality is noticed and in case of more than $1 \mathrm{~cm}$ in the thickness all died.

2) As for the falx sign, in the epidural hematoma with the falx sign the operative mortality was $31 \%$, without the falx sign $40 \%$, however in the subdural hematoma with the falx sign the operative mortality was $50 \%$, in case without falx sign $70 \%$.

3) In anterioposterior view, the more extensive shifting of anterior cerebrat artery developed the higher operative mortality was noted, especially in the subdural hematoma, namely in $1-2 \mathrm{~cm}$ of the shifting contralaterally from the midline mortality was $83 \%$ and in case of more than $2 \mathrm{~cm} 100 \%$. On the other hand in the epidural hematoma in case of $1-2 \mathrm{~cm}$ mortality was $25 \%$ and in more than $2 \mathrm{~cm} 33 \%$.

4) All of intracranial hematoma which A line was lengthen had fatal course, which B line had no relationship to mortality.

5) As for the difference between $\mathrm{H}_{1}$ and $\mathrm{H}_{2}$ in length, in case of more tharr $2.5 \mathrm{~mm}$ the operative mortality was $32 \%$ in the epidural hematoma, and in the subdural hematoma $93 \%$. 
6) As for the intracerebral hematoma, our series are all survived, therefor the clinical consideration was not especially made.

\title{
n-8. Angiographic Study on Traumatic Intracranial Hematoma
}

\author{
Yoshiaki SugIYAMA \\ Department of Neurosurgery, Toyama Prefectural Hospital
}

\begin{abstract}
Noboru Miyazawa, Yukio Kobayashi and Tadashi Tsuchida
Department of Neurosugery, Brain Research Institute, Niigata University
\end{abstract}

Angiographic finding were examined with 123 intracranial hematoma cases encountered in our Clinic for the past four years. Included were fifty-four cases of epidural hematoma, sixty-two cases of subdural hematoma and seven cases of intracerebral hematoma. Most epidural hematoma cases ran acutely and of subdural, chronically. The avascular rea was $n$ angiographic characteristic common to both epidural and subdural hematomas. In Japan some have tried to differentiate epidural hematoma from subdural hematoma by an angiographic finding, i.e., the shape of avascular area in angiogram. Many foreign reports, however, have pointed out that it is difficult to differentiate these two conditions only by the shape of avascular area in angiogram.

Following are the conclusions obtained by an analysis of angiographic findings of 123 cases with intracranial hematoma. In epidural hematoma angiogram analysis, 1) shortness of the interval after head injury and 2) evidence of skull fracture in craniogram should be considered. Taking these two conditions into account, angiographic findnigs obtained from our epidural hematoma cases were similar to those reported by others. We have confirmed that for a correct evaluation of the shape of avascular area it is important to take the angiogram in a condition where the central axis of X-ray runs tangential hematoma. Avascular area was localized and was close to the fracture line. Other angiographic findings of important diagnostic value were extravasation of contrast medium, arteriovenous fistula between the meningeal artery and vein, and the formation of pseudoaneurysm.

In subdural hematoma it is confirmed that 1) in acute stage avascular area was arcuate and covered the whole brain surface unilaterally, 2) in chronic stage it became lenticular and 3 ) in resolving stage it was again arcuate but localized. We experienced one exceptional case in which the angiogram showed extreme displacement of the anterior cerebral artery away from subdural hematoma though epidural hematoma also existed contralateral to subdural hematoma.

Of seven cases of intracerebral hematoma, three cases belonged to type I, four cases to type II and four cases had hematoma in the frontal lobe, three in 